\title{
Effects of Acer okamotoanum sap on the function of polymorphonuclear neutrophilic leukocytes in vitro and in vivo
}

\author{
BEUM-SOO AN $^{1 *}, \mathrm{JI}_{-H O U N} \mathrm{KANG}^{2 *}, \mathrm{HYUN} \mathrm{YANG}^{1}$, MHAN-PYO YANG $^{2}$ and EUI-BAE JEUNG ${ }^{1}$ \\ ${ }^{1}$ Laboratory of Veterinary Biochemistry and Molecular Biology; ${ }^{2}$ Laboratory of Veterinary Internal Medicine, \\ College of Veterinary Medicine, Chungbuk National University, Cheongju, Chungbuk 361-763, Republic of Korea
}

Received August 22, 2012; Accepted November 14, 2012

DOI: $10.3892 / \mathrm{mmr} .2012 .1190$

\begin{abstract}
Sap is a plant fluid that primarily consists of water and small amounts of mineral elements, sugars, hormones and other nutrients. Acer mono (A. mono) is an endemic Korean mono maple which was recently suggested to have health benefits due to its abundant calcium and magnesium ion content. In the present study, we examined the effects of sap from Acer okamotoanum (A. okamotoanum) on the phagocytic response of mouse neutrophils in vivo and rat and canine neutrophils in vitro. We tested the regulation of phagocytic activity, oxidative burst activity (OBA) and the levels of filamentous polymeric actin (F-actin) in the absence and presence of dexamethasone (DEX) in vitro and in vivo. Our results showed that DEX primarily reduced OBA in the mouse neutrophils, and that this was reversed in the presence of the sap. By contrast, the phagocytic activity of the mouse cells was not regulated by either DEX or the sap. Rat and canine polymorphonuclear neutrophilic leukocytes (PMNs) responded in vitro to the sap in a similar manner by increasing OBA. However, regulation of phagocytic activity by the sap was different between the species. In canine PMNs, phagocytic activity was enhanced by the sap at a high dose, while it did not significantly modulate this activity in rat PMNs. These findings suggest that the sap of A. okamotoanum stimulates neutrophil activity in the mouse, rat and canine by increasing OBA in vivo and in vitro, and thus may have a potential antimicrobial effect in the PMNs of patients with infections.
\end{abstract}

Correspondence to: Professor Eui-Bae Jeung, Laboratory of Veterinary Biochemistry and Molecular Biology, College of Veterinary Medicine, Chungbuk National University, 410 SungBong-Ro Heungdeok-gu, Cheongju, Chungbuk 361-763, Republic of Korea E-mail: ebjeung@chungbuk.ac.kr

*Contributed equally

Key words: Acer okamotoanum, polymorphonuclear neutrophilic leukocytes, phagocytosis, oxidative burst activity, dexamethasone

\section{Introduction}

Sap is a plant fluid carried through the xylem cells, which transport water and nutrients in the plant, or through the phloem sieve tube elements (1). Sap primarily consists of water with a small amount of mineral elements, sugars, hormones and other nutrients (1). Acer mono (A. mono) is an endemic Korean mono maple (2) whose sap may be ingested directly as a beverage or concentrated into syrup by boiling it for use as a sweetener $(1,3)$. It has been suggested that the sap of $A$. mono may be beneficial due its abundant calcium and magnesium ion content. A solution of $A$. mono sap ameliorates osteoporosis-like symptoms, and is therefore known as 'bonebenefit water' (1).

Neutrophils, the most abundant type of white blood cells, are phagocytes that play critical roles in combating acute infection and innate immunity (4). These cells have a unique capacity to engulf and eliminate microbes and quickly congregate at the site of infection (5). The phagocytic responses (ingesting microorganisms or particles) of neutrophils against pathogens involve the polymerization and rearrangement of cellular actin filaments (6). The phagocytozed pathogens are then eliminated by microbicidal hydrolytic enzymes and an oxidative burst caused by the formation of reactive oxygen species (ROS) (5). Although increased production of phagocyte-derived ROS may damage host cells and tissues, ROS function as potent antimicrobial agents that protect against infection and cellular signaling molecules under certain conditions (7).

It has been reported that constituents extracted from Acer okamotoanum (A. okamotoanum) leaves and twigs have antioxidant activities (2). However, the effects of the sap on phagocytic responses have not been studied. The objective of the present study was to determine whether the sap of A. okamotoanum affects phagocytic responses of peripheral blood polymorphonuclear neutrophilic leukocytes (PMNs). For this, we performed experiments using mouse PMNs compromised by treatment with dexamethasone (DEX). DEX is a well-known glucocorticoid that impairs the innate functions of phagocytes $(8,9)$. We also examined the in vitro effects of A. okamotoanum sap on phagocytic capacity, oxidative burst activity (OBA) and filamentous polymeric actin (F-actin) levels of mouse PMNs. Our results showed that the 
sap of A. okamotoanum enhanced phagocytic activity and OBA in mouse and canine neutrophils, thus demonstrating its antimicrobial effect.

\section{Materials and methods}

Experimental animals and treatments. Male, 9-week-old ICR mice were obtained from KOATECH (Gyeonggi, Korea). The animals were housed in polycarbonate cages and acclimated in an environmentally controlled room $\left(23 \pm 2^{\circ} \mathrm{C}\right.$; relative humidity, $50 \pm 10 \%$; frequent ventilation; 12 -h light/dark cycle) prior to use. The sap of A. okamotoanum was prepared as previously described (1). To assess the effect of the sap on DEX-treated PMNs, the mice were randomly divided into eight groups. A total of $50 \mathrm{ml}$ tap water or 25,50 or $100 \%$ solutions of sap were administered to the mice at ages 9-16 weeks. Mice in the control group received a physiological saline $(0.9 \% \mathrm{NaCl})$ solution and the other mice (treatment group) received a DEX solution (Sigma-Aldrich Co., St. Louis, MO, USA). Mice in the treatment group received three injections of DEX (1 mg/kg, subcutaneous) every $24 \mathrm{~h}$. For the control group, an equivalent volume of saline solution was injected at the same time points. The experimental procedures were approved by the Ethics Committee of Chungbuk National University (Chungbuk, Korea).

PMN isolation. PMNs were then isolated from rat and canine peripheral blood vessels using a double density gradient centrifugation method to immediately collect blood samples, as described previously $(10,11)$. Briefly, heparinized blood samples were overlaid on a Histopaque-1077 solution (specific gravity, 1.077; Sigma-Aldrich Co.) and Histopaque-1119 solution (specific gravity, 1.199; Sigma-Aldrich Co.) and centrifuged at $700 \mathrm{x}$ g for $40 \mathrm{~min}$ at $20^{\circ} \mathrm{C}$. The PMNs were subsequently harvested from the interface between Histopaque-1077 and Histopaque-1119, and washed three times with cold phosphate-buffered saline (PBS). To purify the PMNs, residual erythrocytes were lysed by treatment with $0.83 \% \mathrm{NH}_{4} \mathrm{Cl}$ in a tri(hydroxymethyl)aminomethane-based buffer ( $\mathrm{pH} 7.2$ ) for $5 \mathrm{~min}$. PMN purity in the final cell suspension was verified to be $>96 \%$ as determined by Wright-Giemsa staining analysis of a blood film obtained by cytocentrifugation. The viability of the PMNs, as determined by trypan blue dye exclusion, was $>97 \%$ in every case. The resulting PMNs were resuspended in RPMI-1640 medium (Sigma-Aldrich Co.) supplemented with $2 \mathrm{mM}$ L-glutamine, $0.02 \mathrm{mg}$ gentamicin $/ \mathrm{ml}$ and $5 \%$ heat-inactivated fetal bovine serum (Invitrogen, Grand Island, NY, USA).

Simultaneous measurement of phagocytic capacity and $O B A$. Phagocytic capacity and OBA were evaluated simultaneously as previously described (12). Briefly, the isolated PMNs were placed in 24-well plates at a density of $1 \times 10^{6}$ cells $/ \mathrm{ml} /$ well and incubated for $2 \mathrm{~h}$ at $37^{\circ} \mathrm{C}$ with $5 \% \mathrm{CO}_{2}$ in a humidified atmosphere. For the in vitro assay, the PMN cells were isolated from rat and canine vessels (abdominal aorta and median antebrachial vein) and incubated with $A$. okamotoanum sap for $2 \mathrm{~h}$ with the previously indicated doses at $37^{\circ} \mathrm{C}$ in a $5 \%$ $\mathrm{CO}_{2}$-humidified atmosphere. A carboxylate-modified polystyrene fluorescent microsphere suspension $(20 \mu \mathrm{l} ; 1.0 \mu \mathrm{m}$ in size; TransFluoSpheres; Molecular Probes Inc., Eugene, OR, USA) adjusted to a concentration of $1 \times 10^{9}$ beads $/ \mathrm{ml}$ was added to the wells for the final $1 \mathrm{~h}$ of culture. When $15 \mathrm{~min}$ of culture time remained, $1 \mu \mathrm{M}$ dihydrorhodamine 123 (Sigma-Aldrich Co.) was added. The conversion of non-fluorescent dihydrorhodamine 123 into fluorescent rhodamine 123 by intracellular ROS was used to measure OBA. The phagocytic capacity was determined by estimating the number of PMNs that had phagocytized fluorescent microspheres in the gated cell population of the sample.

The cultured cells were gently harvested, centrifuged at $400 \mathrm{x}$ g for $3 \mathrm{~min}$ at $4^{\circ} \mathrm{C}$ and washed three times with PBS solution containing $3 \mathrm{mM}$ EDTA. All steps performed following the start of cultivation were conducted in the dark. The cells were analyzed within 30 min using a multipurpose flow cytometer (FACS Calibur system; Becton Dickinson Immunocytometry Systems, San Jose, CA, USA) with an argon laser set at $488 \mathrm{~nm}$ and analysis software (CELLQuest, version 3.3; Becton Dickinson Immunocytometry Systems). The FL1 channel was set to 505-545 $\mathrm{nm}$ to detect green fluorescent rhodamine 123 and the FL3 channel was set to $630-660 \mathrm{~nm}$ to detect red fluorescent microspheres. The cells were gated on the basis of their forward and side light-scattering characteristics. Phagocytic capacity and OBA were expressed as percentages and mean fluorescence intensities (MFIs, arbitrary units), respectively.

Measurement of total cellular F-actin contents. Total cellular F-actin levels were measured as described previously (13). The isolated PMNs were seeded in 24-well plates at a density of $1 \times 10^{6}$ cells $/ \mathrm{ml} /$ well, and then incubated with an extract of $A$. okamotoanum sap for $80 \mathrm{~min}$ at $37^{\circ} \mathrm{C}$ in a $5 \% \mathrm{CO}_{2}$-humidified atmosphere. A carboxylate-modified polystyrene fluorescent microsphere suspension $(20 \mu \mathrm{l} ; 1.0 \mu \mathrm{m}$ in size) adjusted to a concentration of $1 \times 10^{9}$ beads $/ \mathrm{ml}$ was added to the wells for the final $20 \mathrm{~min}$ of culture. The cultured cells were gently harvested, centrifuged at $400 \mathrm{x} \mathrm{g}$ for $3 \mathrm{~min}$ at $4^{\circ} \mathrm{C}$ and washed three times with PBS containing $3 \mathrm{mM}$ EDTA. The viability of the PMNs was verified to be $>98 \%$ based on trypan blue dye exclusion. The cells were fixed with fixation buffer (BD Cytofix; Becton Dickinson Biosciences) at $4^{\circ} \mathrm{C}$ according to the manufacturer's instructions, washed three times with PBS and then stained in the dark for 15 min at $37^{\circ} \mathrm{C}$ with $165 \mathrm{nM}$ FITC-labeled phalloidin (Sigma-Aldrich Co.) and $100 \mu \mathrm{g} / \mathrm{ml}$ lysophosphatidylcholine. The cells were washed and analyzed within $30 \mathrm{~min}$ using a FACS Calibur system (Becton Dickinson Immunocytometry Systems) and analysis software (CELLQuest, version 3.3; Becton Dickinson Immunocytometry Systems) with the argon laser set at $488 \mathrm{~nm}$. Samples from 10,000 cells were assayed in triplicate. The FL1 channel was set to $505-530 \mathrm{~nm}$ to detect the green fluorescing FITC molecule. The F-actin levels were expressed as MFI.

Statistical analyses. The analyses were performed with SigmaStat, version 2.0 (SPSS Inc., Chicago, IL, USA). Differences between the treatment groups were evaluated by a one way analysis of variance (ANOVA), followed by Dunnett's post hoc test. Two-group comparisons were performed with a two-sample t-test. Normality tests (Kolmogorov-Smirnov) were performed to determine whether or not the results had a standard normal distribution. $\mathrm{P}<0.05$ was considered to indicate a statistically significant difference. Results are shown as the mean \pm standard deviation (SD). 
A

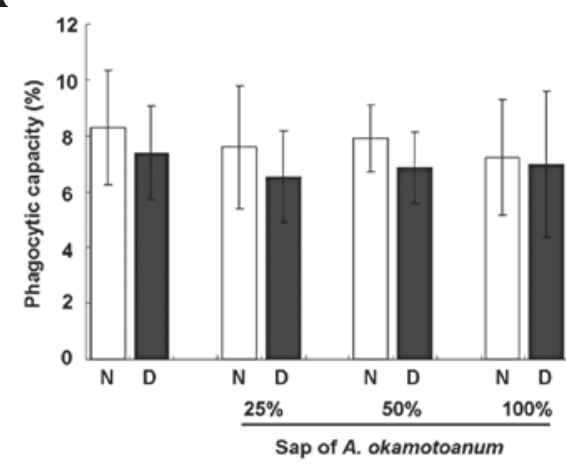

B

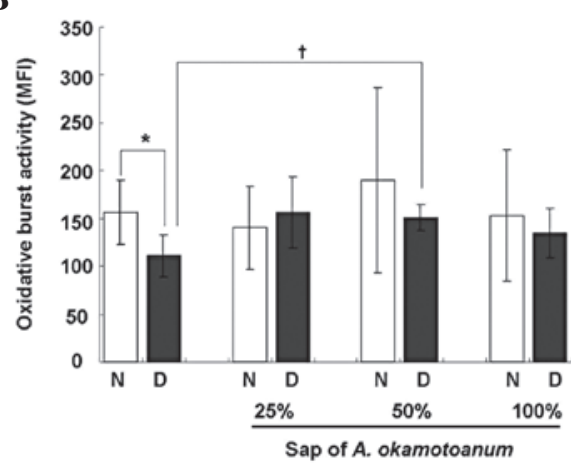

Figure 1. Effect of Acer okamotoanum (A. okamotoanum) sap on (A) phagocytic capacity and (B) OBA of mouse PMNs. Mice were fed A. okamotoanum sap $(0,25,50$ and $100 \%)$ or saline $(0.9 \% \mathrm{NaCl})$ solution (white bars) for 6 weeks in the presence or absence of DEX (1 mg/kg) solution (black bars). Phagocytic capacity and OBA were simultaneously measured from mouse PMNs. "Significant difference $(\mathrm{P}<0.05)$ between the treatment groups; ${ }^{\dagger}$ Significant difference $(\mathrm{P}<0.05)$ vs. the control group without dexamethasone (DEX) treatment. N, no treatment; D, DEX treatment; MFI, mean fluorescence intensity; OBA, oxidative burst activity; PMNs, polymorphonuclear neutrophilic leukocytes.

A

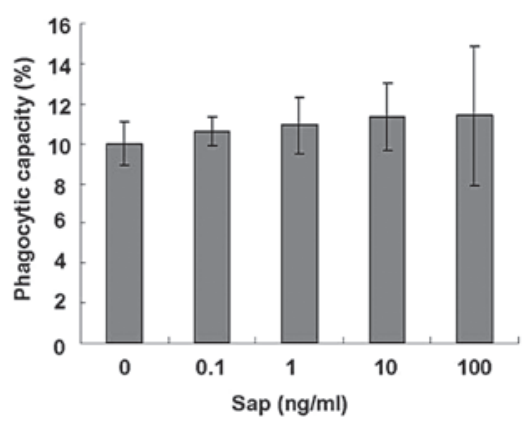

B

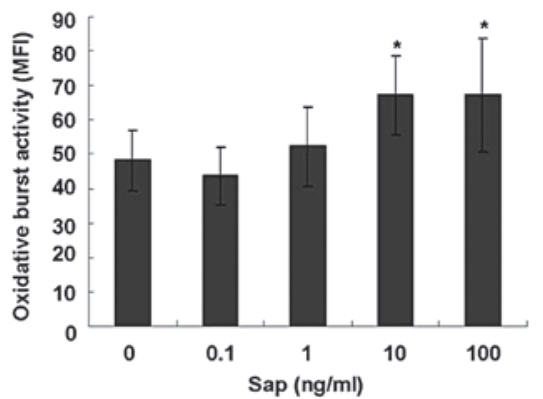

C

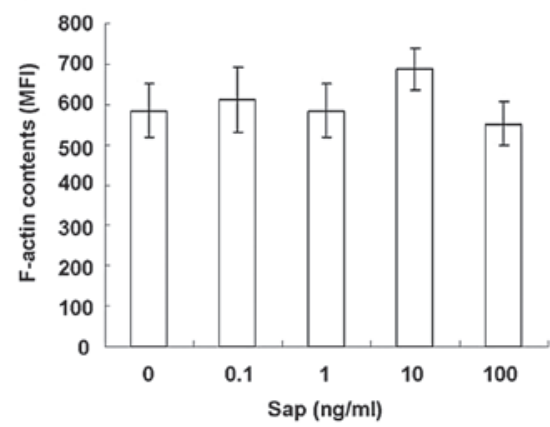

Figure 2. Effects of the Acer okamotoanum (A. okamotoanum) sap extract on (A) phagocytic capacity, (B) OBA and (C) F-actin content (cellular fluorescence intensity) of rat PMNs in vitro. PMNs were isolated from peripheral blood vessels of rats and treated with fluorescent microspheres $\left(2 \times 10^{7}\right.$ beads $/ \mathrm{ml} /$ well $)$ during the final $1 \mathrm{~h}$ of culturing and dihydrorhodamine 123 for the final $15 \mathrm{~min}$. To measure the total cellular F-actin levels, isolated PMNs were cultured for $60 \mathrm{~min}$ and then incubated with fluorescent microspheres $\left(2 \times 10^{7}\right.$ beads/ml/well) for another $20 \mathrm{~min}$. *Significantly different compared with the sap-treated groups $(\mathrm{P}<0.05)$. MFI, mean fluorescence intensity; OBA, oxidative burst activity; PMNs, polymorphonuclear neutrophilic leukocytes.

\section{Results}

Sap of A. okamotoanum reverses DEX-induced reduction of $O B A$ in vivo. To investigate the effect of $A$. okamotoanum sap on phagocytic responses, mature (9-week-old) mice were fed increasing concentrations $(0,25,50$ and $100 \%)$ of A. okamotoanum sap for 6 weeks and were then injected with DEX $(1 \mathrm{mg} / \mathrm{kg})$ for the last 3 days. Following treatment, PMNs were isolated from the mice and the phagocytic capacity and OBA were evaluated. The OBA of the PMNs from mice that received only DEX was significantly decreased $(\mathrm{P}=0.004)$ compared with OBA in cells obtained from the saline-treated group (Fig. 1B). By contrast, the phagocytic capacity of the PMNs was not significantly altered by treatment with DEX $(\mathrm{P}=0.426$; Fig. 1A). The reduction of OBA by DEX was reversed by pretreatment with A. okamotoanum sap at a concentration of $50 \%$, indicating that the sap affected OBA in vivo.

Sap of A. okamotoanum enhances OBA in rat in vitro PMNs. The effects of A. okamotoanum sap on phagocytic capacity, OBA and F-actin content was also determined in vitro using rat PMNs. Similar to the results of the in vivo study, the A. okamotoanum sap extract had no significant effect on the phagocytic capacity ( $\mathrm{P}=0.907$; Fig. $2 \mathrm{~A})$. F-actin content $(\mathrm{P}=0.136$; Fig. $2 \mathrm{C})$ of the rat PMNs was also unaltered by this treatment. However, the OBA of the rat PMNs was increased by treatment with the extract in a dose-dependent manner. These increases were significant at concentrations of 10 and $100 \mathrm{ng} / \mathrm{ml}$ A. okamotoanum sap ( $\mathrm{P}=0.008$; Fig. 2B).

Sap of A. okamotoanum increases phagocytic activity and $O B A$ in canine in vitro PMNs. In addition to rat PMNs, we also examined the effects of A. okamotoanum sap on canine PMNs to determine whether A. okamotoanum sap had antimicrobial activity in other species. We tested various doses $(0.01,0.1,1,10$ and $100 \mathrm{ng} / \mathrm{ml})$ of the sap and found that the effects on phagocytic activity (Fig. 3) were different from that of rat PMNs. The sap increased phagocytic capacity at a dose of $100 \mathrm{ng} / \mathrm{ml}$ in canine PMNs while significant changes were not observed in rat PMNs. The effect on OBA in canine 
A

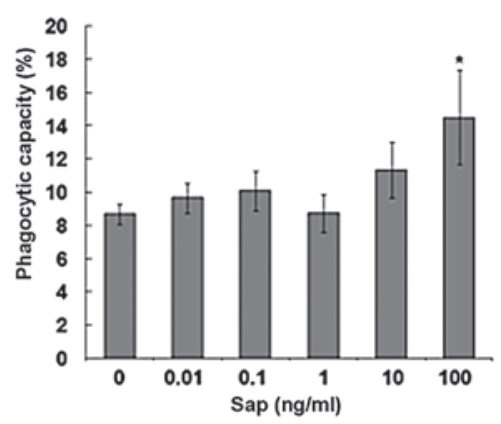

B

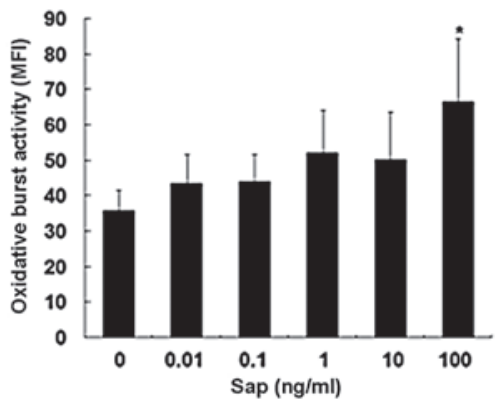

C

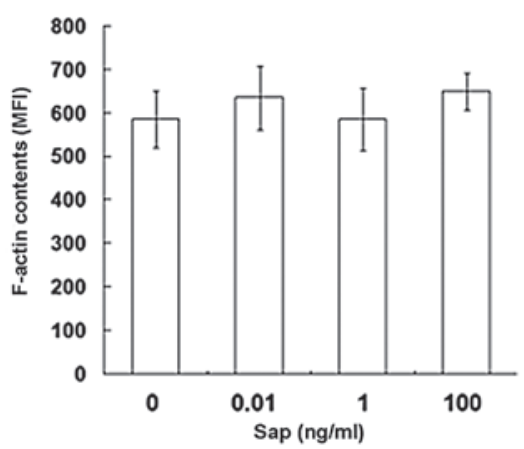

Figure 3. Effects of the Acer okamotoanum (A. okamotoanum) sap extract on (A) phagocytic capacity, (B) OBA and (C) F-actin content (cellular fluorescence intensity) of canine PMNs in vitro. PMNs were isolated from peripheral blood vessels of canines and treated with fluorescent microspheres ( $2 \times 10^{7}$ beads/well) for the final $1 \mathrm{~h}$ of culturing and dihydrorhodamine 123 for the final $15 \mathrm{~min}$. To measure the total cellular F-actin levels, isolated PMNs were cultured for $60 \mathrm{~min}$ and then incubated with fluorescent microspheres ( $2 \times 10^{7}$ beads $/ \mathrm{ml} /$ well) for another $20 \mathrm{~min}$. "Significantly different from those of the sap-treated groups $(\mathrm{P}<0.05)$. MFI, mean fluorescence intensity; OBA, oxidative burst activity; PMNs, polymorphonuclear neutrophilic leukocytes.

PMNs was similar to that observed in rats; this activity was significantly augmented at a high dose of the sap (100 ng/ml). Canine PMN F-actin content, following treatment with the sap at $0.01,1$ and $100 \mathrm{ng} / \mathrm{ml}$ doses, was not significantly altered. These results were similar to those revealed in the rat PMNs (Fig. 3C).

\section{Discussion}

In the present study, we examined the effects of A. okamotoanum sap on the phagocytic response of canine and mouse neutrophils. First, we monitored changes in phagocytic activity, OBA and F-actin contents in the absence and presence of DEX in vivo. Our results showed that DEX reduced OBA and that this effect was reversed in the presence of the sap in mice. The effect of glucocorticoids on the phagocytic responses of phagocytes, including neutrophils, monocytes and macrophages, has been extensively studied, and contradictory results have been reported. Glucocorticoids were found to have an inhibitory effect on ROS production by human monocytes (14), rat peritoneal phagocytes (15) and canine peripheral blood PMNs (13). By contrast, in one study glucocorticoids were reported not to have any effect on the release of reactive oxygen intermediates in cultures of macrophages derived from human blood (16). Another study demonstrated that glucocorticoids increased the phagocytic capacity of human monocytes (14).

To confirm the results of our in vivo study, we performed experiments using rat and canine PMNs. The OBA of rat and canine PMNs was increased by addition of the sap. However, the effects of the sap on phagocytic activity differed between the species. In the canine PMNs, the sap enhanced phagocytic activity at a high dose while it did not have any significant effect on rat PMNs.

Oxidative elimination of microbes is essential for the defense mechanism of neutrophils in the innate immune system (17). This process is accomplished through the generation of ROS by phagocyte nicotinamide adenine dinucleotide phosphate (NADPH) oxidase, and its significance is highlighted in cases of chronic granulomatous disease (18). This disease is characterized by oxidative burst-deficient neutrophils with dysfunctional NADPH oxidase (19).

ROS formation, respiratory burst or neutrophil phagocytosis remove pathogens and thereby offer a defense against these microorganisms. However, the production of free radicals, such as ROS, by activated neutrophils at the site of inflammation also inflicts damage on host tissues. Therefore, regulation of neutrophilic OBA is important for maintaining a balance between host tissue injury and the immune defense during the inflammatory process.

The effects of plant extracts on neutrophil function have previously been reported. For example, Nepeta ucrainica L., a herbal tea, was shown to have a positive effect on respiratory bursts in neutrophils (20). By contrast, other plant extracts, including those from Harpagophytum procumbens, Liriope spicata var. prolifera and apples, are believed to inhibit ROS production, immune responses and inflammation (21-23).

In summary, we determined that treatment with A. okamotoanum sap stimulated the activity of neutrophils from mice, rat and canines by increasing phagocytic activity and OBA in vivo and in vitro. These findings suggest that this sap may have potential antimicrobial effects on the PMNs of patients with infection.

\section{Acknowledgements}

This study was supported by the National Research Foundation of Korea (NRF) grant funded by the Korea government (MEST; no. 2010-0011433).

\section{References}

1. Lee GS, Byun HS, Kim MH, et al: The beneficial effect of the sap of Acer mono in an animal with low-calcium diet-induced osteoporosis-like symptoms. Br J Nutr 100: 1011-1018, 2008.

2. Jin W, Thuong PT, Su ND, et al: Antioxidant activity of cleomiscosins A and C isolated from Acer okamotoanum. Arch Pharm Res 30: 275-281, 2007. 
3. Kunkel G (ed): Plants for Human Consumption: An Annotated Checklist of the Edible Phanerogams and Ferns. 1st edition. Koeltz Scientific Books, Koenigstein, 1984.

4. Segal AW: How neutrophils kill microbes. Annu Rev Immunol 23: 197-223, 2005

5. Gabrilovich DI (ed): The Neutrophils: New Outlook for Old Cells. 1st edition. Imperial College Press, London, 1999.

6. May RC and Machesky LM: Phagocytosis and the actin cytoskeleton. J Cell Sci 114: 1061-1077, 2001.

7. Fialkow L, Wang Y and Downey GP: Reactive oxygen and nitrogen species as signaling molecules regulating neutrophil function. Free Radic Biol Med 42: 153-164, 2007.

8. Long F, Wang YX, Liu L, Zhou J, Cui RY and Jiang CL: Rapid nongenomic inhibitory effects of glucocorticoids on phagocy tosis and superoxide anion production by macrophages. Steroids 70 $55-61,2005$

9. Löwenberg M, Tuynman J, Bilderbeek J, et al: Rapid immunosuppressive effects of glucocorticoids mediated through Lck and Fyn. Blood 106: 1703-1710, 2005.

10. Lee SC, Ju SA, Pack HN, et al: 4-1BB (CD137) is required for rapid clearance of Listeria monocytogenes infection. Infect Immun 73: 5144-5151, 2005.

11. Hasegawa H, Suzuki K, Nakaji S and Sugawara K: Analysis and assessment of the capacity of neutrophils to produce reactive oxygen species in a 96-well microplate format using lucigeninand luminol-dependent chemiluminescence. J Immunol Methods 210: 1-10, 1997.

12. Kang JH and Yang MP: In vitro evaluation of the effect of trans-10, cis-12 conjugated linoleic acid on phagocytosis by canine peripheral blood polymorphonuclear neutrophilic leukocytes exposed to methylprednisolone sodium succinate. Am J Vet Res 69: 494-500, 2008.

13. Kang JH and Yang MP: Effect of a short-term infusion with soybean oil-based lipid emulsion on phagocytic responses of canine peripheral blood polymorphonuclear neutrophilic leukocytes. J Vet Intern Med 22: 1166-1173, 2008.
14. Ehrchen J, Steinmüller L, Barczyk K, et al: Glucocorticoids induce differentiation of a specifically activated, anti-inflammatory subtype of human monocytes. Blood 109: 1265-1274, 2007.

15. Røshol H, Skrede KK, AErø CE and Wiik P: Dexamethasone and methylprednisolone affect rat peritoneal phagocyte chemiluminescence after administration in vivo. Eur J Pharmacol 286: 9-17, 1995.

16. Schaffner A and Schaffner T: Glucocorticoid-induced impairment of macrophage antimicrobial activity: mechanisms and dependence on the state of activation. Rev Infect Dis 9 (Suppl 5): S620-S629, 1987.

17. Roos D, van Bruggen R and Meischl C: Oxidative killing of microbes by neutrophils. Microbes Infect 5: 1307-1315, 2003.

18. Curnutte JT, Whitten DM and Babior BM: Defective superoxide production by granulocytes from patients with chronic granulomatous disease. N Engl J Med 290: 593-597, 1974.

19. Heyworth PG, Cross AR and Curnutte JT: Chronic granulomatous disease. Curr Opin Immunol 15: 578-584, 2003.

20. Akbay P, Calis I, Undeger U, Basaran N and Basaran AA: In vitro immunomodulatory activity of verbascoside from Nepeta ucrainica L. Phytother Res 16: 593-595, 2002.

21. Pastene E, Speisky H, Troncoso M, Alarcón J and Figueroa G: In vitro inhibitory effect of apple peel extract on the growth of Helicobacter pylori and respiratory burst induced on human neutrophils. J Agric Food Chem 57: 7743-7749, 2009.

22. Qi J, Li N, Zhou JH, Yu BY and Qiu SX: Isolation and anti-inflammatory activity evaluation of triterpenoids and a monoterpenoid glycoside from Harpagophytum procumbens. Planta Med 76: 1892-1896, 2010.

23. Hu ZF, Chen LL, Qi J, Wang YH, Zhang H and Yu BY: Two new benzofuran derivatives with anti-inflammatory activity from Liriope spicata var. prolifera. Fitoterapia 82: 190-192, 2011. 